\title{
Selected decays of heavy mesons in covariant confined quark model: Semileptonic and Nonleptonic Decays of $B_{c}$ Meson
}

\author{
A. Liptaj* \\ Institute of Physics, Slovak academy of sciences, Bratislava, Slovakia \\ E-mail: Andrej.Liptaj@savba.sk \\ S. Dubnička \\ Institute of Physics, Slovak academy of sciences, Bratislava, Slovakia
}

A. Z. Dubničková

Faculty of Mathematics, Physics and Informatics, Comenius University, Bratislava, Slovakia

M. A. Ivanov

Joint Institute for Nuclear Research, Dubna, Russia

\section{A. Issadykov}

Joint Institute for Nuclear Research, Dubna, Russia

We use the so-called covariant confined quark model to compute branching fractions for several semileptonic and non-leptonic decay modes of the $B_{c}$ meson.

The European Physical Society Conference on High Energy Physics

5-12 July, 2017

Venice

\footnotetext{
* Speaker.

${ }^{\dagger}$ Support from the Slovak Grant Agency for Sciences VEGA, grant No. 2/0153/17 and from the Slovak Research and Development Agency APVV, grant No. APVV-0463-12, is acknowledged. 


\section{Introduction}

Rare flavor-changing decays of heavy mesons, nowadays measured, are of a special interest: new, hypothetical particles could contribute to the loops of corresponding Feynman diagrams, modify the Standard Model (SM) predictions and so manifest existence of new physics. One source of uncertainty in the theoretical estimation are hadronic effects. Covariant confined quark model (CQM) is a well-suited framework to estimate these and represents a desired alternative for crosschecking existing evaluations of other authors. The CQM is applied to $B_{c}$ semileptonic and nonleptonic decays.

\section{Covariant confined quark model (mesons)}

The covariant confined quark model is meant to provide description of hadronic effects for Standard Model (SM) computations. It is based on a non-local Lagrangian (and thus possesses full Lorentz invariance) where quark-hadron interaction is introduced. The gluons are absent in the model, their effect is taken into account indirectly via vertex functions. The model is suited for description of various multiquark states (mesons, baryons, tetraquarks) and has limited number of free parameters. The interaction Lagrangian is written as

$$
\begin{gathered}
\mathscr{L}_{\text {int }}=g_{M} \cdot M(x) \cdot J_{M}(x), \quad J_{M}(x)=\int d x_{1} \int d x_{2} F_{M}\left(x, x_{1}, x_{2}\right) \cdot \bar{q}_{f_{1}}^{a}\left(x_{1}\right) \Gamma_{M} q_{f_{2}}^{a}\left(x_{2}\right), \\
F_{M}\left(x, x_{1}, x_{2}\right)=\delta\left(x-\omega_{1} x_{1}-\omega_{2} x_{2}\right) \Phi_{M}\left[\left(x_{1}-x_{2}\right)^{2}\right], \quad \omega_{i}=\frac{m_{i}}{m_{1}+m_{2}} .
\end{gathered}
$$

One might mention two important features of the model: compositeness condition and infrared confinement. The compositeness condition (proposed already in the 60ties [1,2]) reflects the fact, that, in nature, hadrons are made of quarks. It states that a state is properly described as bound if it does not contain the "bare" state, i. e. its overlap with it is zero, from which the requirement on the renormalization constant

$$
Z_{M}^{\frac{1}{2}}=0, \text { which translates into CQM as: } 1-\frac{3 g_{M}^{2}}{4 \pi^{2}} \Pi_{M}^{\prime}\left(m_{M}^{2}\right)=0 .
$$

where $\Pi_{M}^{\prime}$ is the derivative of the meson mass operator.

Stability of very heavy mesons is guaranteed by implementation of the infrared confinement. This implementation is related to integration over Schwinger parameters, the latter being used in the quark propagator representation

$$
\widetilde{S}_{q}(k)=(m+\hat{k}) \int_{0}^{\infty} d \alpha e^{\left[-\alpha\left(m^{2}-k^{2}\right)\right]}
$$

The integration region is transformed into a multidimensional simplex convoluted with singe improper integral. Then a single global cutoff parameter $\lambda$ is introduced

$$
\Pi=\int_{0}^{\infty \rightarrow 1 / \lambda^{2}} d t t^{n-1} \int_{0}^{1} d^{n} \alpha \delta\left(1-\sum_{i=1}^{n} \alpha_{i}\right) F\left(t \alpha_{1}, \ldots, t \alpha_{n}\right) .
$$

Numerical integration over Schwinger parameters is used in the computation of transition form factors (being modeled by the CQM) and model-independent amplitudes are evaluated within the helicity formalism. 


\section{Results}

The values of involved Wilson coefficients related to the computation of the results are $a_{1}=$ 1.14 and $a_{2}=-0.20$. The following tables show branching rations as percentages.

\begin{tabular}{|l|l|l|l|l|l|l|l|l|l|l|l|}
\hline Mode & CQM & {$[3]$} & {$[4]$} & {$[5]$} & {$[6]$} & {$[7]$} & {$[8]$} & {$[9]$} & {$[10]$} & {$[11]$} & {$[12]$} \\
\hline$\eta_{c} \ell v$ & 0.98 & 0.81 & 0.98 & 0.75 & 0.97 & 0.59 & 0.15 & 0.40 & 0.76 & 0.51 & 0.44 \\
$\eta_{c} \tau \nu$ & 0.25 & 0.22 & 0.27 & 0.23 & & 0.20 & & & & & 0.14 \\
\hline$J / \psi \ell v$ & 1.74 & 2.07 & 2.30 & 1.9 & 2.35 & 1.20 & 1.47 & 1.21 & 2.01 & 1.44 & 1.01 \\
$J / \psi \tau \nu$ & 0.42 & 0.49 & 0.59 & 0.48 & & 0.34 & & & & & 0.29 \\
\hline $\bar{D}^{-} \ell v$ & 0.0027 & 0.0035 & 0.018 & & 0.004 & 0.006 & & 0.0003 & 0.001 & 0.003 & 0.0032 \\
$\bar{D}^{-} \tau \nu$ & 0.0017 & 0.0021 & 0.0094 & 0.002 & & & & & & & 0.0022 \\
\hline $\bar{D}^{*-} \ell v$ & 0.0050 & 0.0038 & 0.034 & & 0.018 & 0.018 & & 0.008 & 0.008 & 0.013 & 0.011 \\
$\bar{D}^{*-} \tau \nu$ & 0.0028 & 0.0022 & 0.019 & 0.008 & & & & & & & 0.006 \\
\hline
\end{tabular}

\begin{tabular}{|l|l|l|l|l|l|l|l|l|}
\hline Mode & CQM & {$[3]$} & {$[5]$} & {$[6]$} & {$[7]$} & {$[8]$} & {$[10]$} & {$[13]$} \\
\hline$B_{c}^{-} \rightarrow \eta_{c} D_{s}^{-}$ & 0.50 & 0.44 & 0.28 & 0.054 & 0.35 & 0.50 & 0.26 & 1.23 \\
$B_{c}^{-} \rightarrow \eta_{c} D_{s}^{*-}$ & 0.42 & 0.37 & 0.27 & 0.044 & 0.36 & 0.057 & 0.24 & 1.65 \\
$B_{c}^{-} \rightarrow J / \psi D_{s}^{-}$ & 0.22 & 0.34 & 0.17 & 0.041 & 0.12 & 0.35 & 0.15 & 0.81 \\
$B_{c}^{-} \rightarrow J / \psi D_{s}^{*-}$ & 0.78 & 0.97 & 0.67 & & 0.62 & 0.75 & 0.55 & 2.05 \\
\hline$B_{c}^{-} \rightarrow \eta_{c} D^{-}$ & 0.016 & 0.019 & 0.015 & 0.0012 & 0.010 & 0.005 & 0.014 & 0.044 \\
$B_{c}^{-} \rightarrow \eta_{c} D^{*-}$ & 0.019 & 0.019 & 0.010 & 0.0010 & 0.0055 & 0.003 & 0.013 & 0.058 \\
$B_{c}^{-} \rightarrow J / \psi D^{-}$ & 0.0074 & 0.015 & 0.009 & 0.0009 & 0.0044 & 0.013 & 0.009 & 0.028 \\
$B_{c}^{-} \rightarrow J / \psi D^{*-}$ & 0.031 & 0.045 & 0.028 & & 0.010 & 0.023 & 0.028 & 0.067 \\
\hline
\end{tabular}

\section{References}

[1] A. Salam, Nuovo Cim. 25 (1962) 224. doi:10.1007/BF02733330

[2] S. Weinberg, Phys. Rev. 130 (1963) 776. doi:10.1103/PhysRev.130.776

[3] M. A. Ivanov, J. G. Korner and P. Santorelli, Phys. Rev. D 73 (2006) 054024

[4] M. A. Ivanov, J. G. Korner and P. Santorelli, Phys. Rev. D 63 (2001) 074010

[5] V. V. Kiselev, A. E. Kovalsky and A. K. Likhoded, Nucl. Phys. B 585 (2000) 353

[6] C. H. Chang and Y. Q. Chen, Phys. Rev. D 49 (1994) 3399. doi:10.1103/PhysRevD.49.3399

[7] A. Y. Anisimov, I. M. Narodetsky, C. Semay and B. Silvestre-Brac, Phys. Lett. B 452 (1999) 129

[8] P. Colangelo and F. De Fazio, Phys. Rev. D 61 (2000) 034012

[9] D. Ebert, R. N. Faustov and V. O. Galkin, Phys. Rev. D 68 (2003) 094020

[10] A. Abd El-Hady, J. H. Munoz and J. P. Vary, Phys. Rev. D 62 (2000) 014019

[11] M. A. Nobes and R. M. Woloshyn, J. Phys. G 26 (2000) 1079

[12] W. F. Wang, Y. Y. Fan and Z. J. Xiao, Chin. Phys. C 37 (2013) 093102

[13] Z. Rui and Z. T. Zou, Phys. Rev. D 90 (2014) no.11, 114030 